DOI: $10.32743 / f u n . a p p . p r o b l .2021 .33-40$

\title{
Influence of Modification of Aramide Fibers on the Strength of Composite Materials
}

\author{
Tatiana V. BURDIKOVA \\ Ph.D. (in Technical Sciences) \\ Associate Professor \\ Head of the Department
}

Department of Technology of Products from Pyrotechnic and Composite Materials

Kazan National Research Technological University

68, Karl Marx Str., Kazan, 420015, Russia

burdickova@yandex.ru

Lyubov A. ZENITOVA

Ph.D. (in Technical Sciences)

Professor

Head of the Department

Department of Synthetic Rubber Technologies

Kazan National Research Technological University

68, Karl Marx Str., Kazan, 420015, Russia zenit@kstu.ru

\section{Sergey S. IVSHIN}

Ph.D. (in Engineering Sciences)

Associate Professor

Department of Technology of Products from Pyrotechnic and Composite Materials

Kazan National Research Technological University

68, Karl Marx Str., Kazan, 420015, Russia

ivss5555@mail.ru

\author{
Alina A. IVSHINA \\ Assistant \\ Department of Technology of Products from Pyrotechnic and Composite Materials \\ Kazan National Research Technological University \\ 68, Karl Marx Str., Kazan, 420015, Russia \\ lina.ivss@mail.ru
}

This work was financially supported by the Russian Foundation for Basic Research (project no. 18-29-18051).

\begin{abstract}
The paper presents the results of studies to assess the possibility of improving the physical and mechanical characteristics of composite materials due to high-frequency plasma treatment of the surface of aramid fibers. A comparative assessment of the effect of the nature of the plasmaforming gas on the physical and mechanical properties of aramid fibers and composite materials based on them, impregnated with a polyurethane binder based on a prepolymer SKU PFL-100 produced by JSC Kazan Synthetic Rubber Plant, cured with 4,4'-methylene-bis- (orthochloaniline) (MOCA). As a result of the studies carried out, the influence of the parameters of high-frequency plasma treatment on the physical and mechanical characteristics of aramid fibers and composite materials based on them was determined. It is shown that the nature of the plasma-forming gas has a
\end{abstract}


significant effect on the properties of the modified fiber. The highest values of physical and mechanical characteristics were obtained for aramid fibers modified in methane and composite materials based on fibers modified in nitrogen. The effect of the nature of the plasma-forming gas on the physicomechanical characteristics of composite materials based on aramid fibers at negative temperatures has been established.

Keywords: aramid fiber; composite material; low temperature plasma; modification; strength.

\section{Introduction}

One of the achievements of modern chemical technology is the creation of organic aramid fibers. These materials, combining low density (from 970 to $1450 \mathrm{~kg} / \mathrm{m}^{3}$ ), high strength (up to $60 \mathrm{MPa}$ ) and resistance to most corrosive environments, create a large technical and economic effect in a number of industries. The most widely used are aramid fiber materials and composites based on them. Specific strength of organocomposites is 2-3 times higher than titanium and aluminum alloys.

The decisive factor in the formation of the properties of composites is the interaction of the components in the elementary volume of the fiber-matrix. The interaction of the fibers with the matrix should guarantee a high realization of the mechanical properties of the fibers in the reinforced material and its solidity, which is ensured, first of all, by good fiber impregnation with the binder, high adhesion between the fiber and the matrix.

The mechanical behavior of composite materials is determined by the strength of the fibers in the composite, the strength of the bond between the fibers and the matrix, and the plasticity of the matrix. The ratio of these parameters determines the fracture mechanism and the entire complex of mechanical properties of composite materials. With a low bond strength of the fibers with the matrix and sufficiently high values of the other two parameters, the destruction of the composite begins with the violation of the integrity of the interfaces between the components. With low plasticity (embrittlement) of the matrix, cracks in the composite material are transmitted through the matrix and, due to the concentration of stresses, "cut" the fibers encountered in their path, which leads to the destruction of the composition by a single crossing crack. At sufficiently high values of the considered parameters, the appearance of cracks in the composite even with the destruction of weak fibers does not lead to material destruction: the development of microcracks is inhibited by the internal interfaces (matrix-fiber), and the material itself does not lose its bearing capacity (Mikhailov, Fedorov, Fedorova, Vybornov, 2019).

Modification is an effective method for improving the properties of various materials. One of the ways to increase the adhesion strength of fibrous composite materials is to modify the fiber surface. Previous studies have shown that the treatment of organic fibers and fabrics based on them with low-temperature plasma increases the strength of the fibers impregnated with resin (Burdikova, Pavlovets, Meleshko, Roshchin, 2017; Sergeeva, Grishanova, Abdullin, 2010; Sergeeva, Ibatullina, 2012; Sergeeva, Gainutdinova, 2012).

Treatment in low-temperature plasma makes it possible to modify the surface (of a thin surface layer) of a fibrous polymer without changing the bulk properties of the material as a whole. This process is called "plasma modification" and is implemented in three main directions:

- surface activation;

- surface functionalization;

- surface planarization.

The bombardment of the polymer surface with charged plasma particles destroys the covalent bonds between surface atoms and leads to the formation of radicals on the surface. These radicals, in turn, interact with active plasma particles to form various functional groups. At the same time, 
contaminants, weakly bound to the surface, under the influence of energy and chemical factors of the plasma are converted into gaseous compounds and removed from the reactor with a gas flow.

Treatment of a wide range of polymers (polyolefins, polyesters, polyamides, fluoropolymers) in oxygen- and nitrogen-containing plasma for several minutes leads to a significant improvement in the adhesion capacity and hydrophilization of the surface. As a result of treatment, oxygen and nitrogen are introduced into the surface layer with the formation of hydroxyl, carbonyl and carboxyl functional groups. At high oxygen contents in the plasma-forming gas, destruction of some polymers can also occur (chain rupture, etching with the formation of gaseous products). Exposure to a low-pressure high-frequency capacitive (HFC) discharge in an inert gas (argon) leads to the formation of active free radicals, which are converted in air into hydroperoxide and peroxide, and then into stable oxygen-containing polar groups (Mikhailov et al., 2019).

\section{Materials and Methods}

Aramid fibers produced by JSC KAMENSKVOLOKNO were selected as the objects of research in the work. In order to modify the range of fibers with an RFC discharge flow at a reduced pressure, a pilot industrial plasma installation was used (Figure 1). The fibers were processed on a plasmatron at reduced pressures (18-20 Pa), a generator voltage of $900 \mathrm{~kW}$, and a processing time of 5 minutes. After HFC treatment, the fibers were impregnated with a polyurethane binder.

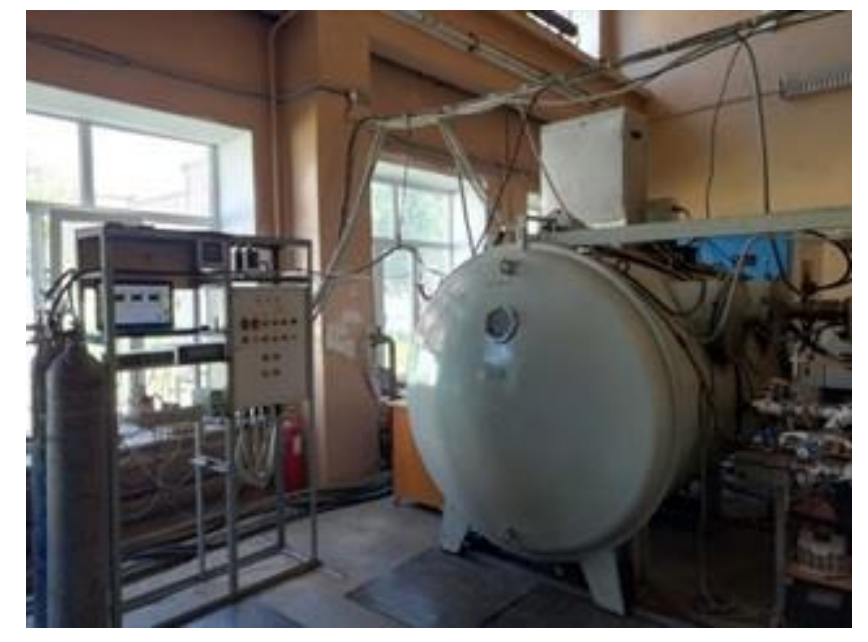

Figure 1. External view of the installation for plasma processing of materials

To obtain a polyurethane binder, a prepolymer SKU PFL-100 (JSC Kazan Synthetic Rubber Plant) and a hardener 4,4'-methylene-bis (orthochloraniline) (MOCA) were used. The prepolymer was preliminarily thermostated at a temperature of $60^{\circ} \mathrm{C}$ for $1 \mathrm{~h}$, after which a hardener was added to it, the amount of which was determined by the following ratio

$$
\mathrm{M}=\frac{\% \mathrm{NCO} \cdot \mathrm{EV} \cdot \mathrm{K}}{42,02}
$$

where: $\mathrm{M}$ is the amount of hardener, mass. hours per 100 mass.h. prepolymer,\% NCO - content of NCO-groups in the prepolymer, EV - equivalent weight of the hardener (for MOCA - 133.5), K stoichiometric coefficient (Burdikova, Zenitova, Ivshin, 2020). The impregnated fibers were cured for 14 days to fully cure the polymer binder. Evaluation of the physical and mechanical properties of aramid fibers was carried out using a universal testing machine "Quasar 100" with a loading speed of $10 \mathrm{~mm} / \mathrm{min}$. For testing under negative temperatures, a climatic chamber connected to a Dewar flask filled with liquid nitrogen was installed in the working area of the testing machine. To assess the wettability of the modified fiber surfaces, the contact angle was determined by the lying drop method (Figure 2) using an Olympus BX43 microscope. 


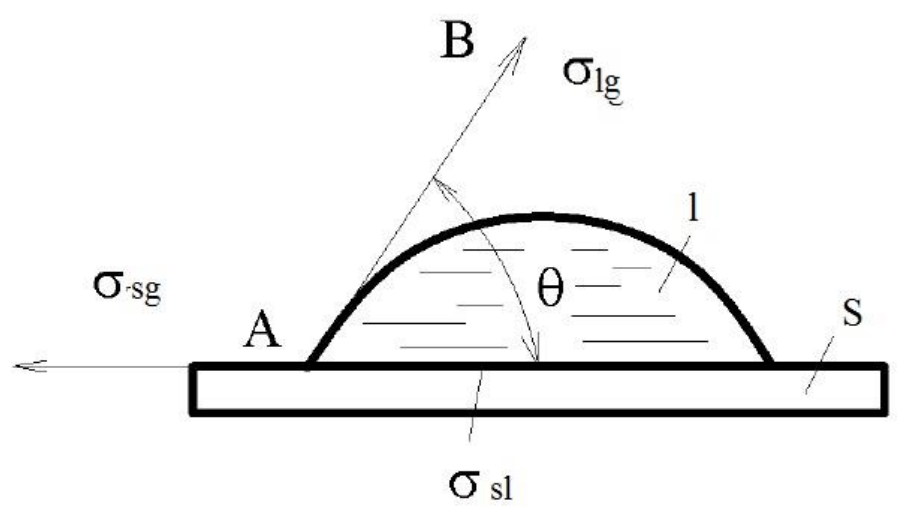

$a$

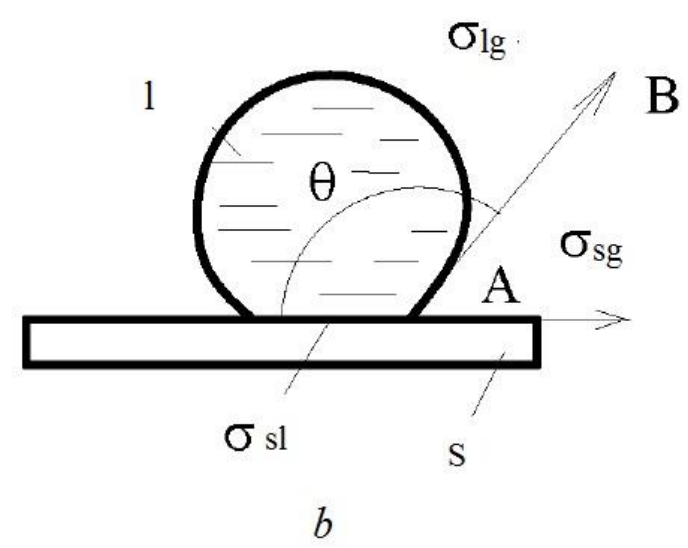

$b$

Figure 2. Contact angle in the system solid - liquid - gas:

\section{a - liquid wets the surface; $b$ - the liquid does not wet the surface}

The angle $\theta$ between the tangent $A B$ to the surface of the liquid and the wetted surface of the solid is called the edge angle. The contact angle is always measured from the tangent towards the fluid. The top of the contact angle $\mathrm{A}$ is located on the wetting line, along which all the phases involved in wetting are in contact (in this case, solid (s), liquid (1), and gas (g)). There are equilibrium and nonequilibrium contact angles. The equilibrium contact angle $\theta$ o depends only on the surface tensions at the interfaces involved in wetting. Therefore, for each system under given external conditions, the equilibrium contact angle has one definite value. The term "contact angle" is used only to characterize the equilibrium state upon wetting. The contact angles measured when the system deviates from the state of thermodynamic equilibrium are called nonequilibrium, or contact $\theta$. Three main cases are distinguished depending on the values of the equilibrium contact angle: 1) nonwetting (poor wetting) - obtuse contact angle $180^{\circ}>\theta>90^{\circ}$; 2) wetting (limited wetting) - acute contact angle $90^{\circ}>\theta>0{ }^{\circ}$; 3) complete wetting - the equilibrium contact angle is not established (the drop spreads out in the form of a thin film). Thus, having determined the contact angle, it is possible to establish the nature of the interaction between a liquid and a solid, and to accurately answer the question of whether there is wetting in the system under study and under these conditions, or there is no wetting. The boundary between wetting and non-wetting is the contact angle $\theta \mathrm{o}=90^{\circ}$. It is generally accepted that at $\theta<90^{\circ}$, liquids wet the surface, and at $\theta>90^{\circ}$, they do not.

\section{Results and Discussion}

The influence of the nature of the plasma-forming gas on the properties of the processed aramid fiber is estimated. The results are shown in Table 1.

Table 1. Influence of the nature of the plasma gas on the physico-mechanical characteristics of the aramid fibers

\begin{tabular}{cccc}
\hline $\begin{array}{c}\text { The nature of the } \\
\text { gas }\end{array}$ & $\sigma_{\text {мax }}, \mathrm{MPa}$ & $\mathrm{E}, \mathrm{MPa}$ & $\varepsilon, \%$ \\
\hline $\begin{array}{c}\text { Without } \\
\text { processing }\end{array}$ & 1352.4 & 12089.2 & 18.8 \\
\hline Air & 1322.1 & 19856.2 & 13.0 \\
\hline Nitrogen & 1282.0 & 19580.4 & 13.9 \\
\hline Argon & 1358.1 & 24314.5 & 13.2 \\
\hline Methane & 1575.9 & 23417.5 & 14.8 \\
\hline
\end{tabular}


The change in the tensile strength of the treated fiber is not so unambiguous. An increase in the strength of aramid fibers was noted for samples modified in methane, which may be associated with the strengthening of the fiber surface layer. A slight increase in strength was also recorded for fiber samples treated in an inert argon atmosphere, which may be due to the healing of surface defects as a result of high-frequency temperature exposure. Nitrogen and oxygen in the air are oxidizing agents, which, apparently, was the reason for the decrease in the tensile strength of the modified aramid fibers. Plasma treatment is a complex complex of physicochemical processes associated with electrical, temperature and chemical effects on the surface, which can cause both the formation of new structures and destructive processes. Probably, the indicated point is a transitional one with a predominance of destructive processes, which is confirmed by microscopic analysis of the surface (Figure 3).

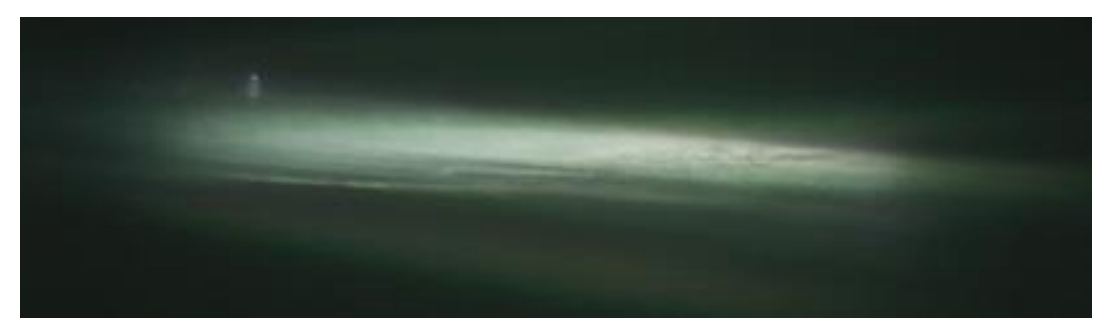

Figure 3. Micrograph of aramid fiber (magnification 500x)

Thus, on the basis of the studies carried out, it has been shown that the treatment of fibers in air and nitrogen gives positive results, namely, an increase in strength by $12 \%$ and an elastic modulus by $10 \%$.

The properties of composite materials based on modified aramid fibers impregnated with a polyurethane binder have been evaluated. The fiber content in the composite was approximately $75 \mathrm{wt} \%$. The test results are shown in Table 2.

Table 2. Physico-mechanical characteristics of composite materials based on modified aramid fibers

\begin{tabular}{lccc}
\hline $\begin{array}{l}\text { The nature of the } \\
\text { gas }\end{array}$ & $\sigma_{\text {мax }}, \mathrm{MPa}$ & $\mathrm{E}, \mathrm{MPa}$ & $\varepsilon, \%$ \\
\hline Without processing & 903.35 & 17079.86 & 12.64 \\
\hline Air & 943.90 & 17198.79 & 11.87 \\
\hline Nitrogen & 1009.97 & 18715.34 & 12.56 \\
\hline Argon & 967.64 & 16057.37 & 12.67 \\
\hline Methane & 965.62 & 16270.50 & 12.93
\end{tabular}

The test results show that the modification of aramid fibers affects the characteristics of composite materials based on it. This reflects the hypotheses put forward about the effect of plasma treatment of the surface layer of the aramid fiber.

The use of nitrogen makes it possible to increase the ultimate strength of the composite material by $18 \%$ while its rigidity remains unchanged; when processing in argon, the rigidity of the material increases by $7 \%$ with a corresponding increase in its ultimate strength. The use of air is associated with the combined effect of nitrogen and oxygen compounds on the fiber surface, which causes fluctuations in material properties within 1\%. At the same time, a decrease in the relative deformation by $6 \%$ is noted.

As a result of the modification, there is an improvement in the wettability of the filler (fiber) surface, which is clearly seen from the change in the contact angle shown in Figure 4. Experimental studies were carried out as follows: on the surface of the fiber located on a solid surface in the 
initial position from a distance of $5 \mathrm{~cm}$ from the dropper a drop of distilled water, the volume of which was $0.2 \mathrm{ml}$. The contact angle was measured during the first 5 seconds according to the standard wetting angle determination method. The data are presented in Table 3 and Figure 4.

Analysis of the results on the contact angle of water droplet wetting applied to the surface of the modified fibers showed that the parameter under study decreases 2-2.5 times compared to the wetting angle of the original fiber.

Table 3. Influence of the nature of the plasma-forming gas on the contact angle of modified fibers

\begin{tabular}{cc}
\hline The nature of the gas & $\begin{array}{c}\text { Contact angle of wetting for 5 s, } \\
\text { degree }\end{array}$ \\
\hline Without processing & 135 \\
\hline Air & 55 \\
\hline Nitrogen & 65 \\
\hline Argon & 60 \\
\hline
\end{tabular}

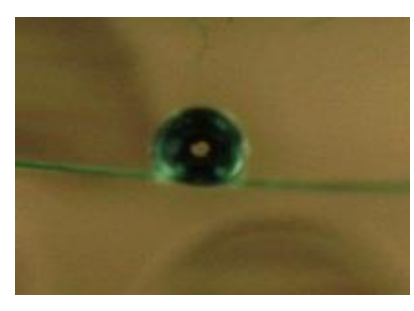

$a$

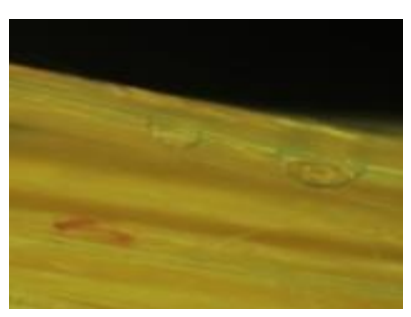

$b$

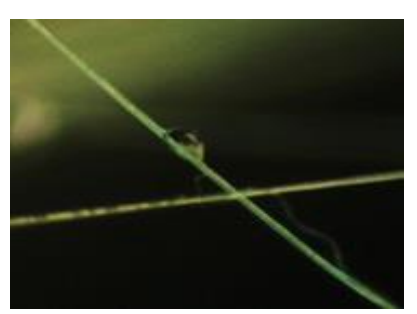

$c$

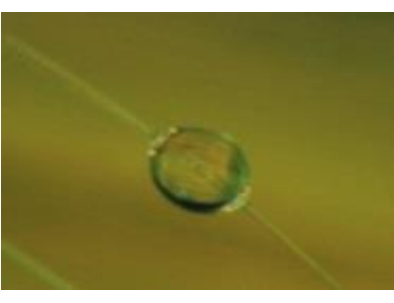

$d$

Figure 4 Micrographs of aramid fibers: $a$ - untreated, $b$ - treated in nitrogen, $c$ - treated in argon, $d$ treated in air (magnification 100x)

The dynamics of changes in the contact angle of wetting was carried out for 45 seconds. Figure 5 shows photomicrographs of modified fibers with water drops applied to their surface, recorded with a step of 15 seconds. It was found that the contact angle of the modified fibers becomes $45^{\circ}$ after 30 seconds, and after 45 seconds they are completely wetted (the contact angle is zero). During this period of time, the contact angle of wetting of a drop of water on the surface of the initial fiber did not change. Studies have shown that the modification of fibers in the environment of the considered plasma gases gives aramid fibers the property of hydrophilicity, which improves the wetting process.

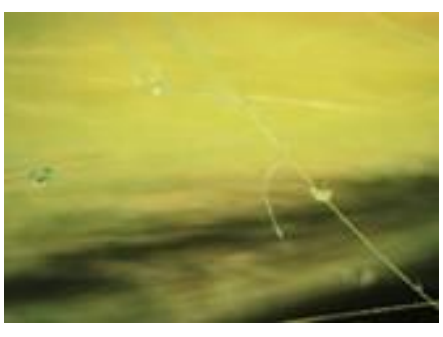

$a$

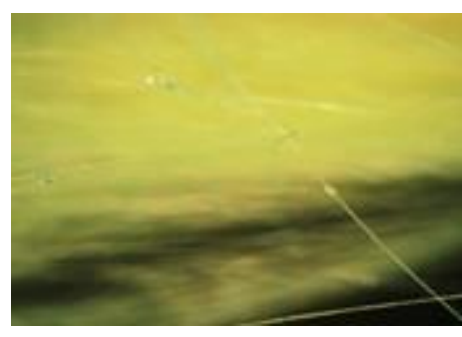

$b$

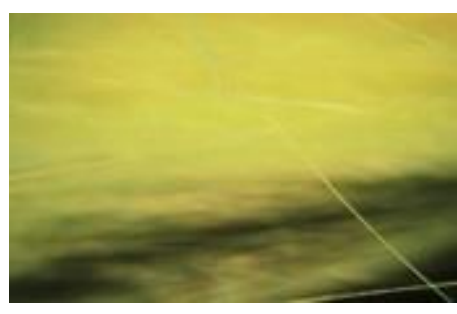

$c$

Figure 5 Dynamics of the process of wetting the treated fibers: $a$ - after 15 seconds, $b$ - after 30 seconds, $c$ - after 45 seconds (50x magnification)

Regardless of the nature of the plasma-forming gas, an increase in the ultimate strength of composite materials based on modified fibers is noted. In this case, an increase in the elastic 
modulus occurs in samples based on aramid fibers modified in nitrogen and air, which is possible in the case of the formation of additional adhesive interactions at the polyurethane-fiber interface. The highest strength values were obtained for fibers treated in a nitrogen environment, which indicates the role of nitrogen-containing groups in the formation of adhesive bonds at the interface.

Since composites filled with aramid fiber are supposed to be used in the Far North, it is advisable to assess the effect of negative temperatures on the properties of polyurethane compositions based on modified aramid fibers.

The test results show that a decrease in the ambient temperature to $-60^{\circ} \mathrm{C}$ leads to a decrease in the relative deformation of the samples, thereby increasing their rigidity and the level of ultimate stresses that the composite material can withstand.

Studies have been carried out to assess the effect of negative temperatures on the characteristics of composite materials based on the original and modified aramid fibers. It was found that a decrease in temperature to $-60^{\circ} \mathrm{C}$ leads to the following changes in the characteristics of the materials under study: for unmodified fibers, an increase in ultimate strength and elastic modulus by $10 \%$, and a decrease in relative deformation by $9 \%$; the use of air as a plasma-forming gas led to an increase in the tensile strength of the materials under study by $9 \%$, the elastic modulus by $11 \%$, and a decrease in the relative deformation by $15 \%$. When the fibers are modified with nitrogen, a decrease also occurs - by $18 \%, 2 \%$ and 5\%; and with argon - by 3\%, 17\% and 9\%, respectively, compared with the results of experiments carried out under normal conditions. It is interesting to note that the treatment of fibers in a nitrogen environment led to an increase in the strength and elastic modulus of the samples, but practically did not reduce the relative deformation at minus $60^{\circ} \mathrm{C}$. This is especially significant, since with increasing strength, the composite remains sufficiently elastic.

Thus, the modification of aramid fibers by plasma treatment makes it possible to regulate the behavior of composite materials based on polyurethane at negative temperatures by changing the nature of the plasma-forming gas.

\section{Conclusion}

As a result of the studies carried out, the influence of high-frequency plasma treatment on the physical and mechanical characteristics of aramid fibers and composite materials based on them was determined. It is shown that the nature of the plasma-forming gas has a significant effect on the properties of the modified fiber. The highest values of the physical and mechanical characteristics of the fibers were obtained for aramid fibers modified in methane, which corresponds to an increase in strength by $16.5 \%$ and an elastic modulus by $93.7 \%$ compared to the original fiber; for composite materials based on fibers modified in nitrogen, which corresponds to an increase in strength by $11.8 \%$ and elastic modulus by $9.6 \%$ compared to the original fiber. The possibility of regulating the behavior of composites under negative temperatures by changing the nature of the plasma-forming gas is shown.

\section{References}

Burdikova, T.V., Pavlovets, G.Ya., Meleshko, V.Yu., Roshchin, A.V. (2017). Influence of plasmachemical modification of reinforcing fibers on the properties of materials functioning under extreme conditions. Technologies and materials for extreme conditions (laser technologies, current sources and materials): collection of articles. materials of reports of participants of the 12th All-Russian scientific conference, 146-153.

Burdikova, T.V., Zenitova, L.A., Ivshin, S.S. (2020). Investigation of the influence of metal nanooxides on the performance characteristics of composite materials based on polyurethanes. Izvestiya vuzov. Chemistry and chemical technology, 63(10), 64-70. 
Mikhailov, A.S., Fedorov, Yu.I., Fedorova, S.Yu., Vybornov, S.A. (2019). Deformation and strength properties of composite and energy-saturated materials. RIC School.

Sergeeva, E.A., Gainutdinova, A.R. (2012). Plasma hydrophilization of aramid tissue for creating ballistic composites. Design. Materials. Technology, 1(21), 80-83.

Sergeeva, E.A., Grishanova, I.A., Abdullin, I.Sh. (2010). Influence of RFC-discharge plasma on physical and mechanical properties of fibers and composite materials. Bulletin of Kazan Technological University, 7, 109-112.

Sergeeva, E.A., Ibatullina, A.R. (2012). Changes in the surface and physical and mechanical properties of aramid fibers modified by a plasma flow of a high-frequency capacitive discharge of low pressure. Bulletin of Kazan Technological University, 4, 63-66. 